\title{
The potential impact of previous exposure to SARS or MERS on control of the COVID-19 pandemic
}

\author{
Junjie Huang ${ }^{1}$. Jeremy Yuen-Chun Teoh ${ }^{2,3} \cdot$ Sunny H. Wong ${ }^{4} \cdot$ Martin C. S. Wong ${ }^{1} \mathbb{C}$
}

Received: 5 June 2020 / Accepted: 4 August 2020 / Published online: 10 August 2020

(c) Springer Nature B.V. 2020

\begin{abstract}
The Severe Acute Respiratory Syndrome coronavirus 2 (SARS-CoV-2) is believed to share similar characteristics with SARS in 2003 and Mediterranean East Respiratory Syndrome (MERS) in 2012. We hypothesized that countries with previous exposure to SARS and MERS were significantly more likely to have fewer cases and deaths from coronavirus disease 2019 (COVID-19). We retrieved the incidence of COVID-19 per 100,000 population within 30 days since the first confirmed case was reported from the 2019 Novel COVID-19 data repository by the Johns Hopkins Centre for Systems Science and Engineering for 94 countries. The association between previous exposure to SARS and/or MERS and the 30-day COVID-19 incidence rate was examined by multivariable linear regression analysis, whilst controlling for potential confounders including the INFORM COVID-19 Risk Index, Testing Policies, Democracy Index, Scientific Citation Index, Gross Domestic Product (GDP), Human Development Index (HDI) and the population density of each country. We found that countries with previous exposure to SARS and/or MERS epidemics were significantly more likely to have lower incidence of COVID-19 ( $\beta$ coefficient $-225.6,95 \%$ C.I. $-415.8,-35.4, p=0.021$ ). However, countries being classified as having "full democracy" using Democracy Index had higher incidence of COVID-19 (reference: authoritarian regime; $\beta$ coefficient 425.0, 95\% C.I. 98.0, 752.0, $p=0.011$ ). This implies that previous exposure to global epidemics and Democracy Index for a country are associated its performance in response to COVID-19. We recommend future studies should evaluate the impact of various pandemic control strategies at individual, community, and policy levels on mitigation of the disease.
\end{abstract}

Keywords Severe acute respiratory syndrome · Mediterranean east respiratory syndrome · Coronavirus disease 2019 . Pandemic control

The coronavirus disease 2019 (COVID-19) has induced a substantial burden to global health in terms of its morbidity and mortality. The Severe Acute Respiratory Syndrome (SARS) in 2003, the Mediterranean East Respiratory Syndrome (MERS) in 2012 and the present COVID-19

Martin C. S. Wong

wong_martin@cuhk.edu.hk

1 Jockey Club School of Public Health and Primary Care, Faculty of Medicine, The Chinese University of Hong Kong, Hong Kong SAR, China

2 Office of Global Engagement, The Chinese University of Hong Kong, Hong Kong SAR, China

3 Department of Surgery, Faculty of Medicine, The Chinese University of Hong Kong, Hong Kong SAR, China

4 Department of Medicine and Therapeutics, Faculty of Medicine, The Chinese University of Hong Kong, Hong Kong SAR, China pandemic in 2019 are caused by the same Betacoronavirus genus-although SARS and MERS are mainly associated with nosocomial spread whilst COVID-19 is transmitted in the community [1]. There is a recent update on the emergence of SARS-like coronavirus in China [2]. Both SARS$\mathrm{CoV}$ and MERS-CoV were believed to originate in bats and were directed transmitted to humans from market civets and dromedary camels, respectively [3]. It was also highlighted that SARS-CoV-2 could display an identity of up to $88 \%$ at the nucleotide sequence level with bat SARS-like CoV genomes [4]. There are a number of significant differences among the three types of SARS-like viruses. When compared with COVID-19, SARS and MERS had much lower transmissibility in terms of their reproductive number, yet their fatality rates are significantly higher. There are, nevertheless, similarities among the three pandemics in terms of their control measures at the country level, including risk communication, isolation strategies, and quarantine policies. 
There has been much discussion regarding whether healthcare policy-makers could learn from previous infectious disease outbreaks such as SARS and MERS-from which the experience might be beneficial to combat the current COVID-19 pandemic. We aimed to evaluate the potential effectiveness of exposure to SARS and/or MERS on incidence of COVID-19 per 100,000 population within 30 days from the first confirmed case reported.

We have previously examined the susceptibility of a country to hazardous incidents and lack of inadequate resources in alleviation of the COVID-19 pandemic based on the INFORM score devised by the European commission [5]. It was found that countries with lower public interest in face mask; a higher COVID-19 vulnerability and lack of coping capacity; as well as those less equipped with certain non-pharmaceutical features [6] were associated with a higher COVID-19 incidence and mortality, after controlling for potential confounding factors. In this study, we retrieved the same incidence outcome as the previous study did [5] from the 2019 Novel COVID-19 data repository by the Johns Hopkins Centre for Systems Science and Engineering (CSSE). A 30-day period was used based on references from published reports. Data on the countries affected by SARS and MERS were extracted from the WHO. We tested the association between the 30-day incidence of COVID-19 and the country-level exposure to SARS and/or MERS, whilst controlling for potential confounders including the INFORM COVID-19 Risk Index, Testing Policies, Democracy Index, Scientific Citation Index, Gross Domestic Product (GDP), Human Development Index (HDI) and the population density. The INFORM COVID-19 Risk Index is a composite score to identify countries at risk from health and humanitarian impacts of COVID-19 that could overwhelm current national response capacity and to support prioritization of preparedness and early response actions for the primary impacts of the pandemic. The COVID-19 testing policies were collected for each country. The Democracy Index in 2019, which evaluated electoral process and pluralism; civil liberties; the functioning of government; political participation; and political culture, was used in the analysis. Scientific Citation Index is defined as the total citations updated to 2019 for each country using data from Scopus. We constructed a multivariable linear regression model with the incidence measure as the outcome variable. We considered $p$ values $<0.05$ as statistically significant. From a literature search of the WHO database, there were 29, 27 and 213 countries or regions affected by SARS, MERS and
COVID-19 at the time of this study, respectively. Figure 1 illustrates the spatial distribution of countries affected by these three pandemics by the world maps. Table 1 shows that countries with previous exposure to SARS and/or MERS epidemics were significantly more likely to have lower incidence of COVID-19 ( $\beta$ coefficient $-225.6,95 \%$ C.I. $-415.8,-35.4, p=0.021)$, after control of potential confounders. However, countries being classified as having "full democracy" using Democracy Index had higher incidence of COVID-19 (reference: authoritarian regime; $\beta$ coefficient $425.0,95 \%$ C.I. 98.0, 752.0, $p=0.011$ ). There was no variable interaction or multicollinearity detected. The leave-oneout cross-validation did not identify any model error driven by a single country for both previous MARS/SARS exposure (Root Mean Squared Errors (MSE): $430.8<469.4$ ) and Democracy Index (Root MSE: $422.9<555.0$ ).

This finding supports that previous exposure to global epidemics and democracy index are associated with the position to respond to COVID-19. Previous pandemic experience might have improved the capabilities to identify and diagnose novel pathogens at a country level, and mitigation strategies might have been implemented more rapidly and effectively to contain the virus. These might include a rapid sharing of the SARS-CoV-2 genetic information; rapid development of diagnostic tests and their implementation; as well as containment and quarantine policies that have mediated a global approach to minimise the spread of COVID-19 by co-coordination of international resources [1]. These findings also encourage national policies to be consolidated by a comprehensive review of effective strategies in this COVID-19 pandemic to enhance population resilience, global health security capacities, surveillance, stringency of containment strategies, isolation measures, and various policies at the individual, community and national levels. A major limitation of the study includes the possible presence of confounders, including number of tests and different short-term strategies, since there is a lack of validated measurement and available data on these factors in all countries. Another limitation is that the cause-and-effect relationships between the exposure to SARS or MERS and the pandemic control of COVID-19 could not be established as a cross-sectional design was adopted in the current study. Future studies that adopt a prospective approach are therefore required to examine the impact of various pandemic control strategies at individual, community, and policy levels on COVID-19 mitigation. 

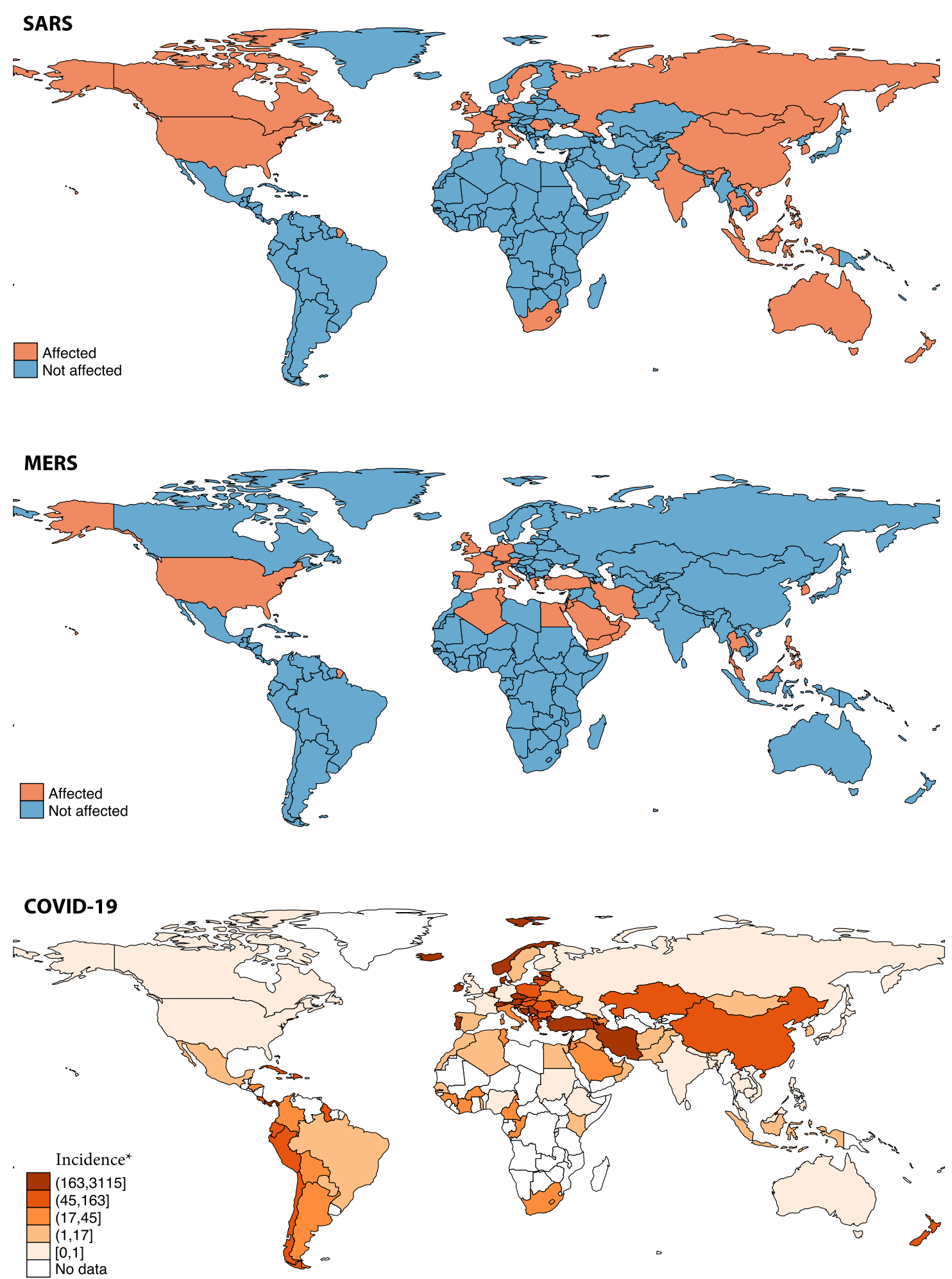

SARS, Severe Acute Respiratory Syndreom; MERS, Mediterranean East Respiratory Syndrome; COVID-19, 2019 coronavirus disease. ${ }^{*}$ Incidence per 100,000 population within 30 days from the report of the first confirmed case.

Fig. 1 Spatial distribution of countries affected by Severe Acute Respiratory Syndrome (SARS), Mediterranean East Respiratory Syndrome (MERS), and 2019 coronavirus disease (COVID-19) 
Table 1 The association between exposure to SARS and/or MERS and the incidence of COVID-19 per 100,000 population within 30 days from the report of the first confirmed case

\begin{tabular}{|c|c|c|}
\hline Epidemics* & & $\begin{array}{l}\text { Number of } \\
\text { countries } \\
\text { affected }\end{array}$ \\
\hline SARS & & 29 \\
\hline MERS & & 27 \\
\hline COVID-19 (as of 05 June, 2020) & & 216 \\
\hline Potential factors associated with incidence & $\beta$ coefficients (95\% C.I.) & $p$ value \\
\hline Never exposure to SARS and/or MERS & Reference & \\
\hline Exposure to SARS and/or MERS & $-225.6(-415.8,-35.4)$ & 0.021 \\
\hline Inform COVID-19 risk index & $66.9(-56.6,190.3)$ & 0.285 \\
\hline Testing policies & $114.8(-42.4,272.0)$ & 0.150 \\
\hline \multicolumn{3}{|l|}{ Democracy index } \\
\hline Authoritarian regime & Reference & \\
\hline Hybrid regime & $-24.0(-314.8,266.9)$ & 0.870 \\
\hline Flawed democracy & $-43.2(-295.8,209.4)$ & 0.735 \\
\hline Full democracy & $425.0(98.0,752.0)$ & 0.011 \\
\hline Scientific citation index & $0.04(-5.14,5.23)$ & 0.987 \\
\hline GDP per capita & $-28.5(-103.9,46.9)$ & 0.454 \\
\hline HDI & $1236.8(-66.1,2539.7)$ & 0.063 \\
\hline Population density & $-20.0(-122.0,82.0)$ & 0.698 \\
\hline
\end{tabular}

*References from: WHO. Summary of probably SARS cases with onset of illness from 1 November 2002 to 31 July 2003. Available at: https:// www.who.int/csr/sars/country/table2004_04_21/en/. Accessed on 05 June, 2020; WHO. Middle East respiratory syndrome coronavirus (MERS$\mathrm{CoV}$ ). Available at: https://www.who.int/emergencies/mers-cov/en/. Accessed on 05 June, 2020; Updated figures from the WHO. Available at: https://www.who.int/emergencies/diseases/novel-coronavirus-2019, Accessed on 05 June, 2020

Acknowledgment We are grateful for the technical assistance offered by Mr. Peter Choi of the School of Public Health and Primary Care, The Chinese University of Hong Kong.

Funding None.

\section{Compliance with ethical standards}

Conflict of interest None declared.

Availability of data and materials Owing to the limits of the reference section, the references for the 2019 Novel COVID-19 data repository by the Johns Hopkins Centre for Systems Science and Engineering (CSSE), INFORM COVID-19 Risk Index, Testing Policies, Democracy Index, Scientific Citation Index, GDP, HDI and population density are listed below, respectively: (1) The 2019 Novel Coronavirus COVID-19 (2019-nCoV) data repository by Johns Hopkins Centre for Systems Science and Engineering (CSSE). Available at: https://systems.jhu.edu/research/public-health/ncov/. Accessed on 05 June, 2020. (2) INFORM COVID-19 Risk Index. Available at: https://drmkc.jrc.ec.europa.eu/inform-index/INFORM-Covid-19. Accessed on 22 July, 2020. (3) COVID-19 Testing Policies. Available at: https://ourworldindata.org/grapher/covid-19-testing-policy. Accessed on 24 July, 2020. (4) Democracy Index 2019. Available at: https://www.eiu.com/topic/democracy-index. Accessed on 14 July, 2020. (5) Scientific Citation Index. Available at: https://www. scimagojr.com/countryrank.php. Accessed on 23 July, 2020. (6) The
Economist Intelligence Unit, World Bank and Central Intelligence Agency World Factbook. Available at: https://www.cia.gov/libra ry/publications/the-world-factbook/geos/we.html. Accessed on 05 June, 2020; (7) 2019 Human Development Index Ranking. United Nations Development Programme, Human Development Reports. Available at: https://hdr.undp.org/en/content/2019-human-devel opment-index-ranking. Accessed on 05 June, 2020. (8) Countries by density by population 2020. World Population Review. Available at: https://worldpopulationreview.com/countries/countries-by-densi ty/. Accessed on 05 June, 2020. A 30-day period was used based on references from published reports (9) Data on the countries affected by SARS and MERS were extracted from the WHO $(10,11)$ listed below: (9). Medium. COVID-19 prevalence and estimated spread in NH. Available at: https://medium.com/less-cancer/covid-19-preva lence-and-estimated-spread-in-nh-bd9876979d5f. Accessed on 05 June, 2020. (10) WHO. Summary of probably SARS cases with onset of illness from 1 November 2002 to 31 July 2003. Available at: https ://www.who.int/csr/sars/country/table2004_04_21/en/. Accessed on 05 June, 2020; (11) WHO. Middle East respiratory syndrome coronavirus (MERS-CoV). Available at: https://www.who.int/emergencie s/mers-cov/en/. Accessed on 05 June, 2020. We have also examined the association between public interest on face mask and COVID19 incidence/mortality, and published the findings at: (12) Sunny $H$ Wong, Jeremy YC Teoh, Chi-Ho Leung, William KK Wu, Benjamin HK Yip, Martin CS Wong, David SC Hui. COVID-19 and public interest in face mask. American Journal of Respiratory and Critical Care Medicine 2020, https://doi.org/10.1164/rccm.202004-1188LE. 


\section{References}

1. Petrosillo N, Viceconte G, Ergonul O, et al. COVID-19, SARS and MERS: are they closely related? Clin Microbiol Infect. 2020;26:729-34.

2. Zhang Z, Xiao K, Zhang X, Roy A, Shen Y. Emergence of SARS-like coronavirus in China: an update. J Infect. 2020;80:e28-9.

3. Cui J, Li F, Shi ZL. Origin and evolution of pathogenic coronaviruses. Nat Rev Microbiol. 2019;17:181-92.

4. Lu R, Zhao X, Li J, Niu P, Yang B, Wu H, et al. Genomic characterisation and epidemiology of 2019 novel coronavirus: implications for virus origins and receptor binding. Lancet. 2020;395:565-74.
5. Wong MC, Teoh JY, Huang J, Wong S. The potential impact of vulnerability and coping capacity on the pandemic control of COVID-19. J Infect. 2020. https://doi.org/10.1016/j. jinf.2020.05.060.

6. Wong MCS, Huang J, Teoh J, Wong SH. Evaluation on different non-pharmaceutical interventions during COVID-19 pandemic: an analysis of 139 countries. J Infect. 2020. https://doi.org/10.1016/j. jinf.2020.06.044.

Publisher's Note Springer Nature remains neutral with regard to jurisdictional claims in published maps and institutional affiliations. 